\title{
Chiral vortical effect in accelerated matter
}

\author{
P. G. Mitkin ${ }^{1}$ and V. I. Zakharov $\circledast^{1,2}$ \\ ${ }^{1}$ ITEP, NRC Kurchatov Institute, 117218 Moscow, Russia \\ ${ }^{2}$ Pacific Quantum Center, Far Eastern Federal University, 690950 Vladivostok, Russia
}

(Received 15 May 2021; accepted 29 November 2021; published 14 December 2021)

\begin{abstract}
We revisit evaluation of the chiral vortical effect in accelerated matter. To first order in the acceleration the corresponding matrix element of the axial current can be reconstructed from its flat-space limit. A crucial point is existence of an extra conservation law of fluid helicity which is not related to the symmetry of the Lagrangian. As a result, one can reproduce, via the equivalence principle, the effect of the so-called gravimagnetic anomaly; resolving in this way a long standing puzzle of its interpretation. Moreover, as a consequence of the extra conservation law the microscopic axial charge and helicity of the macroscopic motion are separately conserved. Some further consequences from the matching of the equivalence principle with hydrodynamics concerning higher orders in gradient expansion or in the acceleration are briefly discussed.
\end{abstract}

DOI: 10.1103/PhysRevD.104.125011

\section{INTRODUCTION}

Chiral effects have attracted a lot of interest recently. These effects can influence the dynamics of a variety of systems, from the interior of a neutron star and quark-gluon plasma to Weyl and Dirac semimetals (for a review see, e.g., $[1,2])$. Originally these effects were evaluated in the case of heated fermionic gas in the presence of rotation and/or an external magnetic field [3-5]. More recently, it was realized that in the case of ideal fluid the chiral effects can be related to the quantum chiral anomaly $[6,7]$ and the corresponding transport coefficients are explicitly calculable.

In absence of electromagnetic fields, the axial current $j_{5}^{\mu}$ receives a vortical contribution (the chiral vortical effect, or CVE),

$$
j_{5}^{\mu}=n_{5} u^{\mu}+\sigma_{\omega} \omega^{\mu}
$$

where $n_{5}$ is the density of the microscopic constituents with axial charge, $u_{\mu}$ is the four-velocity of an element of the fluid, satisfying $u^{2}=-1$, and $\omega^{\mu}=\frac{1}{2} \epsilon^{\mu \nu \alpha \beta} u_{\nu} \partial_{\alpha} u_{\beta}$ is the vorticity. The vortical conductivity $\sigma_{\omega}$ is directly related to the coefficient $C_{5}$ in front of the anomaly:

$$
\partial_{\mu} j_{5}^{\mu}=C_{5} \boldsymbol{E} \cdot \boldsymbol{B} \rightarrow \sigma_{\omega}=\mu^{2} C_{5}+\frac{c T^{2}}{6} .
$$

Published by the American Physical Society under the terms of the Creative Commons Attribution 4.0 International license. Further distribution of this work must maintain attribution to the author(s) and the published article's title, journal citation, and DOI. Funded by SCOAP.
Here $\mu$ is the chemical potential and $T$ is the temperature of the system. For a single Dirac fermion $C_{5}=\frac{1}{2 \pi^{2}}$ and $c=1$.

The $T^{2}$ term in the rhs of the Eq. (2) was obtained first within the standard thermal field theory [8], but the discussion on its origin is still going on, see e.g., [9-18]. In particular, it is suggested to be related to the gravitational anomaly:

$$
\nabla_{\mu} j_{5}^{\mu}=-\frac{1}{384 \pi^{2}} \frac{\epsilon^{\mu \nu \rho \sigma}}{\sqrt{-g}} R_{\beta \mu \nu}^{\alpha} R_{\alpha \rho \sigma}^{\beta}
$$

where $R_{\alpha \beta \gamma \delta}$ is the Riemann tensor. This relation is hard to check in the hydrodynamic approach. Indeed, the righthand side of (3) is of the fourth order in gradients and the corresponding current would be of the third order, while the CVE is of the first order, see (1). It was suggested, however, to interpret the generic temperature $T$ as the Unruh temperature $T_{U}=a /(2 \pi)$ where $a$ is the gravitational acceleration (for details see $[13,15,16]$ ). This substitution eliminates two of the gradients of the gravitational field in the hydrodynamic picture and allows to link the $T^{2}$ part of the CVE to the anomaly (3).

In addition to the gravitational anomaly (3), there is another specific contribution to the divergence of the axial current in the external gravitational field (see [19] and references therein). Namely, it is argued that simultaneous account for the rotation and the acceleration results in the so-called gravimagnetic anomaly. The argumentation is based on the well-known analogy between rotation and the magnetic field. In more detail, one introduces the gravimagnetic fields $\boldsymbol{E}_{g}=-\epsilon \boldsymbol{\nabla} \varphi, \boldsymbol{B}_{g}=2 \epsilon \boldsymbol{\Omega}$, where $\varphi$ is the Newtonian potential, $-\boldsymbol{\nabla} \varphi=\boldsymbol{a}_{g}$, and $\epsilon$ is an energy of an interacting test particle. Substituting this into (2) instead of 
the usual electromagnetic fields and summing over the Fermi sphere one gets, see [19] and references therein:

$$
\partial_{\mu} j_{5}^{\mu}=\frac{\mu^{2}}{\pi^{2}} \boldsymbol{a}_{g} \cdot \boldsymbol{\Omega}
$$

Note that the rhs of (4) is of the second order in gradients. Clarifying the physical meaning of (4) is one of our aims here.

As is known, the chiral anomaly (2) reveals instabilities in the chiral media with a nonzero axial chemical potential $\mu_{5} \neq 0$, which is closely connected with the existence of additional conservation laws of macroscopic helicities [20-31]. Namely, a state with $Q_{5} \neq 0$ decays into a state with a helical magnetic field. Generally speaking, both of the anomalies [(3) and (4)] can trigger similar phenomena. In particular, on the basis of (4) one could argue that the state with a nonvanishing microscopic chirality $Q_{5}$ can be transformed into a helical macroscopic motion, even in the absence of the electromagnetic interactions. If true, this would be a highly nontrivial theoretical phenomenon.

Studying the CVE in an external gravitational field is also interesting for two other reasons. First, the phenomenology of heavy ion collisions indicates that the matter produced in these experiments undergoes accelerated expansion and that this acceleration cannot be treated as a small gradient correction (see [32] and further references in [2]). Another point of interest in turning on gravitation is that it is known to mimic a temperature gradient [33],

$$
a_{g} \rightarrow-\frac{\nabla T}{T}
$$

The advantage of exploiting this relation is that the acceleration $\boldsymbol{a}_{g}$ can be introduced within a well-understood field-theoretic framework, while the notion of the temperature belongs to thermodynamics.

In this paper, we start with a review of the conservation laws of an ideal chiral fluid in a flat spacetime, and discuss the nature of these laws in Sec. II. In Sec. III we turn to hydrodynamics in the presence of a weak external gravitational field. We evaluate the terms linear in the gravitational acceleration following the standard procedure, see, e.g., [34]. Namely, we start with the conservation laws in the absence of an external gravitational field and then evaluate their modification by gravity. Following this procedure, we show that Eq. (4) is simply a manifestation of the equivalence principle and actually coincides with Eq. (3) in the absence of the $R \tilde{R}$ term. In the same section we identify a relation between the expansion coefficients at the second order in gradients. In Sec. IV we briefly discuss the terms of higher order in acceleration. Throughout the text we use the metric signature $(-,+,+,+)$, and the normalization of the Levi-Civita symbol as $\epsilon^{0123}=\sqrt{-g}$.

\section{CONSERVATION LAWS FOR IDEAL FLUIDS}

Let us first consider an ideal fluid in the absence of gravity. The hydrodynamics represent a universal approach based on the conservation laws and expansion in gradients.

It is known, however, that in the case of an ideal fluid there exists extra conservation laws, not related to the symmetries of the Lagrangian (see, e.g., [20,24]). The simplest example of such a law is the conservation of the magnetic helicity:

$\mathcal{H}_{m}=\frac{1}{4 \pi^{2}} \int d^{3} x \boldsymbol{A} \cdot \boldsymbol{B}, \quad \frac{d \mathcal{H}_{m}}{d t}=-\frac{1}{2 \pi^{2}} \int d^{3} x \boldsymbol{E} \cdot \boldsymbol{B}$.

If the chemical potential is constant, the electric field is screened inside an ideal conductor, and the magnetic helicity is conserved. Indeed, for an electric current $\boldsymbol{J}_{e l}=$ $\sigma_{e} \boldsymbol{E}$ to be finite in the limit of the perfect magnetohydrodynamics $\sigma_{e} \rightarrow \infty$ the electric field inside the fluid should vanish [35].

Let us emphasize that this conservation law is purely dynamical and is not related to a symmetry of the Lagrangian. Rather, this physics is sensitive to boundary conditions [36]. This is a generic difference between the specific conservation laws of an ideal fluid and Noether currents which are conserved locally. Potentially, existence of the extra conservation laws is a source of an unusual infrared sensitivity. We will come back to discuss this point later.

Chiral magnetic and chiral vortical effects are closely related to the extra conservation laws. Indeed, with account for the quantum chiral anomaly the conserved axial charge for a system of massless fermions is given by

$$
Q_{5}=Q_{5}^{\text {naive }}+\mathcal{H}_{m}
$$

where $Q_{5}^{\text {naive }}$ is a naive microscopic axial charge.

In the effective field theory describing the electromagnetic interaction of fermions in the hydrodynamic approximation [39], the effective interaction of the microscopic degrees of freedom with a photon field is shifted due to the chemical potential

$$
A_{\mu} \rightarrow A_{\mu}+\mu u_{\mu}
$$

If we substitute this into (7), we get two additional hydrodynamic contributions to the axial charge, one being the fluid helicity $\mathcal{H}_{f}=\frac{1}{2 \pi^{2}} \int d^{3} x \mu^{2} \omega^{0}$, and the other being the fluid-magnetic helicity $\mathcal{H}_{m f}=\frac{1}{2 \pi^{2}} \int d^{3} x \mu B^{0}$, with $B^{\mu}=$ $\frac{1}{2} \epsilon^{\mu \nu \alpha \beta} u_{\nu} F_{\alpha \beta}$ being the magnetic field in the comoving frame of the element of fluid. Let us note that this substitution does not capture the $T^{2}$ part of the vortical effect. With account for that part, the total axial charge is given by 


$$
Q_{5}=Q_{5}^{\text {naive }}+\mathcal{H}_{m}+\mathcal{H}_{f}+\mathcal{H}_{m f}+\mathcal{H}_{t f},
$$

where the magnetic, fluid, and fluid-magnetic helicities can be associated with the currents

$$
j_{m}^{\mu}=\frac{1}{4 \pi^{2}} \epsilon^{\mu \nu \alpha \beta} A_{\nu} \partial_{\alpha} A_{\beta}, \quad j_{f}^{\mu}=\frac{1}{2 \pi^{2}} \mu^{2} \omega^{\mu}, \quad j_{m f}^{\mu}=\frac{1}{2 \pi^{2}} \mu B^{\mu},
$$

while the thermal fluid helicity $\mathcal{H}_{t f}$ is the charge corresponding to

$$
j_{t f}^{\mu}=\frac{1}{6} T^{2} \omega^{\mu}
$$

Such an analysis, based on the consideration of hydrodynamic charges rather than spatial currents, albeit not new, is generally unnoted in the literature. Moreover, quantum field theory brings in a novel perspective on the role of the extra conservation laws.

Indeed, the substitution (8) demonstrates the connection between the chiral vortical conductivity and the quantum anomaly existing even in the absence of electromagnetic fields. A crucial point is that the connection holds for the calculation of the currents, not their divergences. The reason is that the anomalous contributions to the currents are defined by the infrared properties of the system, while the divergences of anomalous currents are defined by the physics in the ultraviolet. Roughly speaking, if we try to reconstruct currents knowing their divergences the contributions discussed appear as "integration constants." In other words, the condition of vanishing divergences of the novel contributions to the currents becomes a constraint on the equation of state of the ideal fluid.

Let us elaborate this point following [24]. The condition $\sigma_{e l} \rightarrow \infty$ requires $\frac{E_{\alpha}}{T}-\partial_{\alpha} \frac{\mu}{T}=0$. Introducing a kinematic acceleration $a_{\mu}=u^{\rho} \partial_{\rho} u_{\mu}=u^{\rho} \omega_{\rho \mu}$, with $\omega_{\rho \mu}=\partial_{\rho} u_{\mu}-\partial_{\mu} u_{\rho}$, and algebraically decomposing $\omega_{\mu \nu}$ in $a_{\mu}$ and $\omega_{\mu}$ one finds

$$
\partial_{\mu} \omega^{\mu}=2 a_{\mu} \omega^{\mu}
$$

Note that Eq. (12) is purely kinematic. In order to account for the dynamics of the fluid we use the relativistic Euler equation,

$$
a_{\alpha}=-\frac{1}{\epsilon+p} \Delta_{\alpha}^{\beta} \partial_{\beta} p
$$

where $\epsilon$ is the energy density, $p$ is the pressure, and the projector $\Delta_{\alpha \beta}=g_{\alpha \beta}+u_{\alpha} u_{\beta}$ satisfies $u^{\alpha} \Delta_{\alpha \beta}=0$. Using that along with thermodynamical relations $d p=n d \mu+s d T$ and $\epsilon+p=n \mu+s T$ (here $n$ is the density of the chosen kind of particles and $s$ is the entropy density), one can rewrite (13) as

$$
a_{\alpha}=-\Delta_{\alpha}^{\beta}\left(\frac{\partial_{\beta} T}{T}+\frac{n T}{\epsilon+p} \partial_{\beta}\left(\frac{\mu}{T}\right)\right) .
$$

It is convenient at that point to change the thermodynamic variables from $\mu, T$ to $\frac{\mu}{T}$ and $T$. Then for the various parts of the conserved hydrodynamic current we have

$2 \pi^{2} \partial_{\alpha} j_{f}^{\alpha}=2 \omega^{\alpha}\left(\frac{T^{2} s \mu}{\epsilon+p} \partial_{\alpha}\left(\frac{\mu}{T}\right)+\frac{\mu^{2} n T}{\epsilon+p} \frac{E_{\alpha}}{T}\right)=2 \mu E_{\alpha} \omega^{\alpha} ;$

$$
\begin{aligned}
2 \pi^{2} \partial_{\alpha} j_{m f}^{\alpha} & =\left(\partial_{\alpha} \mu-\mu a_{\alpha}\right) B^{\alpha}-2 \mu E_{\alpha} \omega^{\alpha}, \\
& =E_{\alpha} B^{\alpha}-2 \mu E_{\alpha} \omega^{\alpha} .
\end{aligned}
$$

We see that the sum of the magnetic, fluid, and mixed helicities is conserved by itself,

$$
\partial_{\alpha} j_{m}^{\alpha}+\partial_{\alpha} j_{f}^{\alpha}+\partial_{\alpha} j_{m f}^{\alpha}=0
$$

which means that there is no production of the microscopic axial charge in the ideal-fluid regime and

$$
\partial_{\alpha}\left(n_{5} u^{\alpha}\right)=0
$$

even though the macroscopic helicities can interchange with each other. Let us note that this picture breaks with the introduction of viscosity but as long as the ideal-fluid approximation is valid, it stays true.

As for the $T^{2}$ contribution, it is simply conserved by itself:

$$
\partial_{\alpha}\left(T^{2} \omega^{\alpha}\right)=\frac{2 n T^{3}}{\epsilon+p}\left(\partial_{\alpha}\left(\frac{\mu}{T}\right)-\frac{E_{\alpha}}{T}\right) \omega^{\alpha}=0 .
$$

In particular, it seems that, in the ideal regime, there is no mechanism of transition between this part of fluid vorticity and the magnetic helicity, although vortical response in the photonic helical current is nonzero [11].

Note that all of these additional conservation laws take place only in the stationary case. This brings up the question of what happens if we introduce some nonstationarity into the system. One way to do that is to consider the system in an external gravitational field, which is the focus of the next section. As was discussed in the Introduction, it seems that in this case there should be some additional inputs to the hydrodynamic charge. Another possible source of nonconservation is dissipative effects, which, in turn, can translate the nonstationarity caused by an external field into a stationary flow, which is characterized by the corresponding transport coefficients. In principal, there should be some corrections to the hydrodynamic charges in higher orders in the gradient expansion as well. 


\section{TURNING ON GRAVITY}

Now we consider the case of a weak external gravitational field and study the terms which are linear in gravity. In particular, we focus on the gravimagnetic anomaly, which itself is linear in the acceleration.

The central point of our argument is that we can use the flat-space conservation laws and rewrite them via the equivalence principle to include the leading gravitational effects. For instance, in the absence of electromagnetic fields we can write the fluid helicity conservation as

$$
\frac{1}{2 \pi^{2}} \nabla_{\alpha} \mu^{2} \omega^{\alpha}=\frac{1}{2 \pi^{2} \sqrt{-g}} \partial_{\alpha}\left(\sqrt{-g} \mu^{2} \omega^{\alpha}\right)=0,
$$

where $\nabla_{\alpha}$ is the covariant derivative. However, in the weak gravitational field approximation we can rewrite it as a correction to its flat-background form, $\partial_{\mu} \omega^{\mu}=0$. To do so let us consider an ideal fluid in an external weak gravitational field, $h_{\mu \nu}=g_{\mu \nu}-\eta_{\mu \nu}$, of a simple form with the only nonzero component being

$$
h_{00}=-2 b \text {, }
$$

where $b=b(t, \boldsymbol{x})$ plays the role of a gravitational potential. If, in addition to the condition of the gravitational field to be weak, we require the fluctuations of the velocity of the fluid to be small as well, $u^{i}=v^{i} \ll 1$, then the four-velocity of the fluid takes the form $u^{\mu}=(1-b, v)$. Substituting this into Eq. (20) and noticing that $\sqrt{-g}=1+b+\mathcal{O}\left(h^{2}\right)$, we get

$$
\partial_{\alpha} j_{f,(0)}^{\alpha}=-\frac{\mu^{2}}{\pi^{2}}(\boldsymbol{\Omega} \cdot \boldsymbol{\nabla} b),
$$

with $\boldsymbol{\Omega}=\frac{1}{2} \boldsymbol{\nabla} \times \boldsymbol{v}$.

Turning to the relativistic case we notice that the metric in form (21) says nothing about the state of the fluid in the geometry described by that metric. In fact, choice (21) actually fixes the frame we are working in without any reference to the fluid flow. In general, one has to specify the boundary conditions in order to proceed. For our purposes we have to describe a fluid such that each of its elements is accelerating. That means that the metric should have form (21) in the local rest frame of a fluid element, and we use the following ansatz:

$$
h_{\mu \nu}=-2 b u_{\mu} u_{\nu},
$$

with $b=-\frac{1}{2} u^{\mu} u^{\nu} h_{\mu \nu}$.

Let us stress that the redefinition (23), while looking harmless, is in fact a nontrivial procedure beyond the first order in gradients. Unlike the decomposition $F_{\mu \nu}=E_{\mu} u_{\nu}-$ $E_{\nu} u_{\mu}+\epsilon_{\mu \nu \alpha \beta} u^{\alpha} B^{\beta}$, which is identical and simply serves as a definition of $E_{\mu}$ and $B_{\mu}$, in the general case Eq. (23) genuinely makes $h_{\mu \nu} u$-dependent and in that sense resembles the chemical shift (8). Much like the latter, which in the case of a uniform flow is simply a $U(1)$ gauge transformation, this decomposition makes the gravitational field gauge nontrivial as $u_{\mu}$ becomes dynamic.

To preserve some notion of stationarity, or, to be more specific, "comoving" stationarity, we also require that

$$
u^{\mu} \partial_{\mu} b=0 \text {. }
$$

Now we can proceed to expand the hydrodynamic variables in $h_{\mu \nu}$. We will denote the order in $h_{\mu \nu}$ by the subscript/superscript (i). First let us note that a change in the geometry modifies the four-velocity of the flow, which we can write as a series in $h_{\mu \nu}: u^{\mu}=u_{(0)}^{\mu}+u_{(1)}^{\mu}+\ldots$. The function $u_{(0)}^{\mu}$ would then play the role of a "flat" fluid velocity. The corrections of the first order can be easily found from the condition $g_{\mu \nu} u^{\mu} u^{\nu}=-1$ :

$$
u_{\mu}=u_{\mu}^{(0)}(1+b)+\mathcal{O}\left(h^{2}\right) .
$$

Now we can use that and the equivalence principle to find the divergence of the zero-order currents. Starting first with a naive current $n_{5} u^{\mu}$, one can see that in the ideal regime in a flat space this current is conserved, and therefore

$0=\frac{1}{\sqrt{-g}} \partial_{\mu} \sqrt{-g} n_{5} u^{\mu}=\frac{1}{\sqrt{-g}} \partial_{\mu} n_{5} u_{(0)}^{\mu}+\mathcal{O}\left(h^{2}\right)$.

Thus, we see that the usual divergence of the naive current is zero.

Proceeding further to the other equations of the ideal hydrodynamics, we first consider the fluid helicity in the external gravitational field,

$$
\partial_{\alpha} j_{f,(0)}^{\alpha}=-\frac{\mu^{2}}{\pi^{2}} \omega_{(0)}^{\alpha} \partial_{\alpha} b .
$$

We see that this equation actually saturates the gravimagnetic anomaly (4), and coincides with Eq. (3). Note that we do not refer at all to the chiral gravitational anomaly but keep only the terms with the gravitational field coming from the Christoffel symbols and the definition of the fourvelocity on a curved background.

One can also demonstrate that this result is actually independent of the observer's rotation. To see that we can simply introduce an intrinsic rotation in the rest frame via a new parameter in the metric as $h_{\mu \nu}=-2 b u_{\mu} u_{\nu}-$ $V_{\mu} u_{\nu}-V_{\nu} u_{\mu}$, and fixing the gauge with $V_{\mu} u^{\mu}=0$, $u^{\mu} \partial_{\nu} V_{\mu}=0$. Equation (27) then reads

$$
\partial_{\alpha} j_{f,(0)}^{\alpha}=-\frac{\mu^{2}}{\pi^{2}}\left(\omega_{(0)}^{\alpha}-\Omega^{\alpha}\right) \partial_{\alpha} b,
$$


where $\Omega^{\alpha}=\frac{1}{2} \epsilon^{\alpha \beta \mu \nu} u_{\beta}^{(0)} \partial_{\mu} V_{\nu}$ can be thought of as an intrinsic angular velocity of the observer's frame. This equation explicitly shows that the gravimagnetic anomaly, much like the CVE itself (see [40] for further discussion), is induced specifically by the fluid rotation and in this sense it is frameindependent.

Coming back to the case $V_{\mu}=0$, and including the effects of an external electromagnetic field, we find

$$
\begin{gathered}
\partial_{\alpha} j_{t f,(0)}^{\alpha}=-\frac{T^{2}}{3} \omega_{(0)}^{\alpha} \partial_{\alpha} b \\
\partial_{\alpha} j_{f,(0)}^{\alpha}+\partial_{\alpha} j_{m f,(0)}^{\alpha}+\partial_{\alpha} j_{m,(0)}^{\alpha} \\
=-\left(\frac{\mu}{2 \pi^{2}} B_{(0)}^{\alpha}+\frac{\mu^{2}}{\pi^{2}} \omega_{(0)}^{\alpha}\right) \partial_{\alpha} b .
\end{gathered}
$$

All of these equations are a consequence of the equivalence principle and can be arrived at starting from the flat case through the substitution

$$
a_{\text {kinematic }}=a_{\text {gravity }}
$$

where $a_{\text {gravity }}^{\mu}=-\partial^{\mu} b$.

The equivalence principle also allows us to study the thermal effects using the analogy between the temperature gradient and the gravitational field [33] through the Luttinger substitution,

$$
\partial_{\mu} b=-a_{\mu}^{g}=\frac{\partial_{\mu} T}{T} .
$$

Since the terms linear in $\partial_{\mu} b$ are fixed by the equivalence principle, we expect that the terms linear in the $\frac{\partial_{\mu} T}{T}$ should be fixed as well. This conjecture can be used to determine the relation between the different transport coefficients. We should note, however, that the substitution (32) should be used cautiously as it depends on the frame we are working in. In particular, the stationarity condition (24) implies that the four-velocity satisfies

$$
\frac{1}{T} u^{\mu} \partial_{\mu} T=0
$$

which is a nontrivial condition.

To illustrate this point we turn to a nondissipative effect reminiscent of a thermal Hall effect, and recently discussed in [41]. Namely, we consider two second-order contributions to the electric current (a current of a conserved charge) of the form

$$
\delta j^{\mu}=c_{1} \Delta_{\rho}^{\mu} \partial_{\nu} \omega_{\perp}^{\nu \rho}-\frac{c_{2}}{T^{2}} \omega_{\perp}^{\mu \nu} \partial_{\nu} T,
$$

where $\omega_{\perp}^{\mu \nu}$ is a part of $\omega^{\mu \nu}$ that is orthogonal to $u^{\mu}$,

$$
\omega_{\perp}^{\mu \nu}=\frac{1}{2}\left(\partial_{\perp}^{\mu} u^{\nu}-\partial_{\perp}^{\nu} u^{\mu}\right),
$$

with $\partial_{\perp}^{\mu} u^{\nu}=\Delta^{\mu \rho} \partial_{\rho} u^{\nu}=\partial^{\mu} u^{\nu}+u^{\mu} a^{\nu}$, so that

$$
\omega_{\perp}^{\mu \nu}=\omega^{\mu \nu}+\frac{1}{2} u^{\mu} a^{\nu}-\frac{1}{2} a^{\mu} u^{\nu}=-\epsilon^{\mu \nu \alpha \beta} u_{\alpha} \omega_{\beta} .
$$

In the simplest case of a conformal theory, dimensional analysis tells us that $c_{1}=T f_{1}\left(\frac{\mu}{T}\right)$ and $c_{2}=T^{2} f_{2}\left(\frac{\mu}{T}\right)$. Since the second term in Eq. (34) is linear in the temperature gradient, we expect that it could be recovered from the covariant form of the first term in (34) in the external gravitational field via substitution (32). However, in the case of a conformal theory one can argue that the effective description of the thermal gradients in terms of gravitational fields should preserve the conformal symmetry, i.e., the metric (23) should be replaced by a conformal metric, $g_{\mu \nu}=(1+2 b) \eta_{\mu \nu}$. Note that this redefinition does not change the four-velocity rescaling (25), as it is defined only by the projection of the metric on the velocity itself ( $g_{00}$ in the comoving rest frame), and, therefore, the coefficients of the gravimagnetic anomaly stay the same. However, the expected contribution in (34) changes. Indeed, we have $\omega_{\mu}=\omega_{\mu}^{(0)}+\mathcal{O}\left(b^{2}\right)$, and

$$
\begin{aligned}
\delta j^{\mu} & =c_{1} \nabla_{\nu} \Delta_{\rho}^{\mu} \omega_{\perp}^{\nu \rho}=c_{1} \Delta_{\rho}^{\mu} \frac{1}{\sqrt{-g}} \partial_{\nu}\left(\sqrt{-g} \omega_{\perp}^{\nu \rho}\right) \\
& =(1-3 b) c_{1} \Delta_{\rho}^{\mu} \partial_{\nu} \omega_{\perp,(0)}^{\nu \rho}-c_{1} \omega_{\perp,(0)}^{\mu \nu} \partial_{\mu} b .
\end{aligned}
$$

Thus, the first term in (34) results in the second term under the Luttinger substitution, and we find that

$$
c_{2}=T c_{1},
$$

in agreement with [41]. Notice that the Luttinger substitution relates only the acceleration and the temperature gradient but the metric components gain no new meaning. The extra term in (37) coming with $b$ without gradients can be removed if we turn off the external gravitational field after the second term of (34) is identified.

\section{MATCHING THE EQUIVALENCE PRINCIPLE AND THERMODYNAMICS}

So far, while considering the fluid in the external gravitational field, we focus on the case when the gravity introduces nonstationarity into the picture. This, again, partially fixes the boundary conditions in our problem. Another approach is to consider an accelerating fluid in the equilibrium. To do that one must include acceleration into parameters characterizing matter in the equilibrium. The corresponding density operator $\hat{\rho}$ relevant to this case was worked out rather recently (see [42]): 


$$
\hat{\rho}=\frac{1}{Z} \exp \left(-\beta_{\alpha} \hat{P}^{\alpha}-\frac{1}{2} \varpi_{\alpha \beta} \hat{J}^{\alpha \beta}\right),
$$

where $\hat{P}^{\alpha}$ is the momentum operator, $\hat{J}^{\alpha \beta}$ are generators of the Lorentz rotations, $\beta_{\alpha}=\frac{u_{\alpha}}{T}$, and $\varpi_{\alpha \beta}=\frac{1}{2}\left(\partial_{\alpha} \beta_{\beta}-\partial_{\beta} \beta_{\alpha}\right)$ is the thermal vorticity tensor. Note that, in contrast with $\omega_{\alpha \beta}$, which includes only the kinematic acceleration and vorticity of the fluid, $\varpi_{\alpha \beta}$ also depends on the gradients of the temperature. The Lorentz generators in (39) can be decomposed into the boost operators $\hat{K}$ and generators of rotations $\hat{J}$ :

$$
\hat{J}^{\alpha \beta}=u^{\alpha} \hat{K}^{\beta}-u^{\beta} \hat{K}^{\alpha}-\epsilon^{\alpha \beta \gamma \delta} u_{\gamma} \hat{J}_{\delta} .
$$

Knowing the density operator $\hat{\rho}$ one can evaluate oneloop statistically averaged matrix elements, such as matrix elements of the energy-momentum tensor $T^{\alpha \beta}$ or of the axial current $J_{5}^{\alpha}$, see in particular $[43,44]$. The novel point is that the boost operators are conserved but do not commute with the Hamiltonian [42]. If one does not introduce the vorticity, then it turns out that the matrix elements are polynomials in the acceleration $a$ and temperature $T$. The whole calculation is in terms of the Minkowskian space, and the acceleration $a$ is now what we call kinematic acceleration, $a_{\text {kinematic }}$.

On the other hand, one can evaluate the same matrix elements associated with particles living on a nontrivial manifold with a boundary-a so-called conical manifoldat a finite temperature. The acceleration is then introduced in geometrical terms and represents the gravitational acceleration.

It was demonstrated that, say, energy density $\rho_{s=1 / 2}$ for massless spin- $1 / 2$ particles is exactly the same within the both approaches, statistical and gravitational:

$$
\rho_{s=1 / 2}=\frac{7 \pi^{2} T^{4}}{60}+\frac{T^{2} a^{2}}{24}-\frac{17 a^{4}}{960 \pi^{2}} .
$$

This coincidence of the results can be considered a confirmation of the equivalence principle in a nonlinear form. For further details see the original paper [44].

\section{DISCUSSION}

In these notes we have considered an application of the equivalence principle to the ideal fluid hydrodynamics. From the very beginning, we put an emphasis on the conservation laws inherent to ideal fluids, since the conservation laws are the backbone of the hydrodynamic approach. Considering the fluid in the ideal regime allows us to use the formalism of unitary field theories. Since we are interested in hydrodynamics we chose an effective field theory which describes physics of equilibrium. The corresponding effective vertices can readily be read off from the form of the density operator.
Simple manipulations within the effective field theory allow us to re-establish a fundamental observation: that in ideal hydrodynamics there are extra conservation laws which are highly nonlocal and not related to the symmetries of the Lagrangian - the conservation laws of macroscopic helicities. In fact, these conservation laws have been discussed for a long time in terms of classical theory. An unexpected turn of logic is that now they get related to the quantum anomalies of the underlying field theory. While the anomaly is determined by the short-distance physics, the helical currents emerge as an infrared completion of the theory.

We utilize the equivalence principle to consider the accelerated motion of the fluid. The main point of this discussion is that it can be used to obtain the effects linear in gravitational acceleration. A particular effect that can be recovered this way is the so-called gravimagnetic anomaly. Formally, this result coincides with the result of Ref. [19]. The interpretation is, however, different. From our point of view, the consequences of the equivalence principle reduce to a classical observation $a_{\text {kinematic }}=a_{\text {gravity }}$, where $a_{\text {kinematic }}$ is associated with the introduction of a noninertial coordinate system, while $a_{\text {gravity }}$ is the acceleration induced by an external gravitational field. The gravimagnetic anomaly appears not to be associated with any violation of the chiral symmetry.

Although in our case [45] application of the equivalence principle results in a routine answer, we encounter a kind of fundamental problem that the introduction of the gravitational acceleration brings in nonstationarity. This particular issue becomes especially interesting if we go in another direction following [33]. If we introduce dissipative effects, this nonstationarity translates into a (stationary) transport phenomenon. The nonstationarity that external field brings in is important for this: say, in the stationary rotating fluid there should be a temperature gradient causing the centrifugal force but no transport along this gradient. This becomes especially apparent if we go to the reference frame rotating with a fluid, where the temperature gradient is screened by a gravitational (inertial) force. In general the correct statement is that the dissipative transport is caused by the fields that are not screened in the "comoving" coordinate maps (see, for example, $[48,49]$ ). This in turn leads us to a conjecture that a global comoving coordinate map exists in general only for an ideal fluid and dissipative effects are in this sense similar to the introduction of a genuine gravitational field. This also indicates the specific nature of the conservation laws we are working with. For the ideal fluid, every fundamental conservation law should exist separately for each fluid element, and when the gravitational field (which itself should be invariant with respect to the coordinate diffeomorphisms) is applied, each volume element should behave independently. Conservation of the macroscopic fluid helicities, however, depends on the boundary conditions and does not fit into this picture. For now we leave this topic for a future discussion. 
We also illustrate a broader applicability of the equivalence principle in hydrodynamics, showing that some higher order responses involving the temperature gradient can be related to the other transports. Such relations help to construct a consistent hydrodynamic theory at higher orders, and may be essential for phenomenological applications. We leave the further considerations of such related transport coefficients for future work.

Finally, in Sec. IV we review the recent developments in the descriptions of the accelerating-fluid equilibrium. For that one can include the kinematic acceleration of the fluid into the density operator $\hat{\rho}$ (39) and use it to calculate statistically averaged matrix elements [42], or do the same calculations on a curved background. The coincidence of the results obtained using these methods can serve as an additional confirmation of the equivalence principle. Our final remark is that within the statistical approach linear in acceleration terms are absent. Indeed, these terms do not contribute to the matrix elements, see $[42,44]$. At first sight, this result is in conflict with our procedure to fix the nonzero linear terms starting from the equivalence principle. However, there is no contradiction between the two approaches since they are applied to two different regimes, equilibrium and nonequilibrium. Moreover, comparison of these two types of calculations makes manifested dependence of the linear in acceleration terms on physics in far-infrared, which we have emphasized in the preceding sections.

\section{ACKNOWLEDGMENTS}

The authors are thankful to O. V. Teryaev and A.V. Sadofyev for detailed discussions. The work on this paper has been partly supported by RFBR Grants No. 18-0240056 and No. 0657-2020-0015 of the Ministry of Science and Higher Education of Russia. The work of P. M. is also supported by the Foundation for the Advancement of Theoretical Physics and Mathematics "BASIS" No. 20$1-5-134-1$.
[1] X.-G. Huang, Rep. Prog. Phys. 79, 076302 (2016).

[2] D. Kharzeev, J. Liao, S. Voloshin, and G. Wang, Prog. Part. Nucl. Phys. 88, 1 (2016).

[3] A. Vilenkin, Phys. Rev. D 20, 1807 (1979).

[4] A. Vilenkin, Phys. Rev. D 22, 3080 (1980).

[5] A. Vilenkin, Phys. Rev. D 21, 2260 (1980).

[6] J. Erdmenger, M. Haack, M. Kaminski, and A. Yarom, J. High Energy Phys. 01 (2009) 055.

[7] D. T. Son and P. Surowka, Phys. Rev. Lett. 103, 191601 (2009).

[8] A. Vilenkin, Phys. Rev. D 20, 1807 (1979).

[9] K. Landsteiner, E. Megias, and F. Pena-Benitez, Phys. Rev. Lett. 107, 021601 (2011).

[10] S. Golkar and D. T. Son, J. High Energy Phys. 02 (2015) 169.

[11] A. Avkhadiev and A. V. Sadofyev, Phys. Rev. D 96, 045015 (2017).

[12] P. Glorioso, H. Liu, and S. Rajagopal, J. High Energy Phys. 01 (2019) 043.

[13] A. Flachi and K. Fukushima, Phys. Rev. D 98, 096011 (2018).

[14] K. Landsteiner, E. Megias, L. Melgar, and F. Pena-Benitez, J. High Energy Phys. 09 (2011) 121.

[15] M. Stone and J. Kim, Phys. Rev. D 98, 025012 (2018).

[16] G. Yu. Prokhorov, O. V. Teryaev, and V. I. Zakharov, Phys. Rev. D 102, 121702 (2020).

[17] X.-G. Huang, P. Mitkin, A. V. Sadofyev, and E. Speranza, J. High Energy Phys. 10 (2020) 117.

[18] A. Alexandrov and P. Mitkin, J. High Energy Phys. 05 (2021) 070.

[19] G. Basar, D. E. Kharzeev, and I. Zahed, Phys. Rev. Lett. 111, 161601 (2013).

[20] J. D. Bekenstein, Astrophys. J. 319, 207 (1987).
[21] Y. Akamatsu and N. Yamamoto, Phys. Rev. Lett. 111, 052002 (2013).

[22] Z. V. Khaidukov, V. P. Kirilin, A. V. Sadofyev, and V. I. Zakharov, Nucl. Phys. B934, 521 (2018).

[23] V. P. Kirilin, A. V. Sadofyev, and V. I. Zakharov, in Proceedings of the 100th Anniversary of the Birth of I. Ya. Pomeranchuk (Pomeranchuk 100): Moscow, Russia, June 5-6, 2013 (World Scientific, Singapore, 2014), pp. 272-286.

[24] A. Avdoshkin, V. Kirilin, A. Sadofyev, and V. Zakharov, Phys. Lett. B 755, 1 (2016).

[25] C. Manuel and J. M. Torres-Rincon, Phys. Rev. D 92, 074018 (2015).

[26] P. V. Buividovich and M. V. Ulybyshev, Phys. Rev. D 94, 025009 (2016).

[27] N. Yamamoto, Phys. Rev. D 93, 065017 (2016).

[28] Y. Hirono, D. Kharzeev, and Y. Yin, Phys. Rev. D 92, 125031 (2015).

[29] V. P. Kirilin and A. V. Sadofyev, Phys. Rev. D 96, 016019 (2017).

[30] Y. Li and K. Tuchin, Phys. Lett. B 776, 270 (2018).

[31] K. Tuchin, Phys. Rev. C 102, 014908 (2020).

[32] B. Abelev et al. (STAR Collaboration), Phys. Rev. C 76, 024915 (2007); 95, 039906(E) (2017).

[33] J. Luttinger, Phys. Rev. 135, A1505 (1964).

[34] S. M. Carroll, arXiv:gr-qc/9712019.

[35] Note that the results for the chiral vortical effect (central for this paper) also survive if one introduces a finite resistance. What is crucial, however, is to stick to the approximation $\eta=0$. The limit $\sigma_{e} \rightarrow \infty$ is an extra assumption, common in considerations of the magnetic helicity (see, for example, [20]), but not relevant to discussions of fluid helicity and CVE. 
[36] Notice that chiral media support multiple boundary effects $[37,38]$, which could additionally complicate the boundary conditions.

[37] K. Fukushima, S. Imaki, and Z. Qiu, Phys. Rev. D 100, 045013 (2019).

[38] M. B. Farias, A. A. Zyuzin, and T. L. Schmidt, Phys. Rev. B 101, 235446 (2020).

[39] A. Sadofyev, V. Shevchenko, and V. Zakharov, Phys. Rev. D 83, 105025 (2011).

[40] Y.-C. Liu, L.-L. Gao, K. Mameda, and X.-G. Huang, Phys. Rev. D 99, 085014 (2019).

[41] S. Li, M. A. Stephanov, and H.-U. Yee, Phys. Rev. Lett. 127, 082302 (2021).

[42] F. Becattini, Phys. Rev. D 97, 085013 (2018).
[43] G. Prokhorov, O. Teryaev, and V. Zakharov, Phys. Rev. D 98, 071901 (2018).

[44] G. Y. Prokhorov, O. V. Teryaev, and V. I. Zakharov, J. High Energy Phys. 03 (2020) 137.

[45] There exist examples $[46,47]$ when the standard program of renormalization at finite temperature comes in contradiction with the equivalence principle.

[46] J. F. Donoghue, B. R. Holstein, and R. W. Robinett, Gen. Relativ. Gravit. 17, 207 (1985).

[47] M. Buzzegoli and D. E. Kharzeev, Phys. Rev. D 103, 116005 (2021).

[48] S. Dubovsky, L. Hui, A. Nicolis, and D. T. Son, Phys. Rev. D 85, 085029 (2012).

[49] P. Glorioso and D. T. Son, arXiv:1811.04879. 